\section{Spectroscopic analysis of the oxygenation state of hemoglobin using coherent anti-Stokes Raman scattering}

\author{
Hilde A. Rinia, ${ }^{\text {a }}$ Mischa Bonn, ${ }^{\mathrm{b}}$ Erik M. Vartiainen, ${ }^{\mathrm{c}}$ \\ Chris B. Schaffer, ${ }^{d}$ and Michiel Müller ${ }^{a, *}$ \\ anniversity of Amsterdam, Swammerdam Institute for Life \\ Sciences, P.O. Box 94062, 1090 GB Amsterdam, \\ The Netherlands \\ ${ }^{\mathrm{b}} \mathrm{FOM}$ Institute for Atomic and Molecular Physics (AMOLF), \\ Kruislaan 407, 1098 SJ Amsterdam, The Netherlands \\ 'Lappeenranta University of Technology (LUT), Department \\ of Electrical Engineering, Box 20, Fin-53851 \\ Lappeenranta, Finland \\ ${ }^{\mathrm{d}}$ Cornell University, Department of Biomedical Engineering, \\ 120 Olin Hall, Ithaca, New York 14853
}

Abstract. A method for noninvasively determining blood oxygenation in individual vessels inside bulk tissue would provide a powerful tool for biomedical research. We explore the potential of coherent anti-Stokes Raman scattering (CARS) spectroscopy to provide this capability. Using the multiplex CARS approach, we measure the vibrational spectrum in hemoglobin solutions as a function of the oxygenation state and observe a clear dependence of the spectral shape on oxygenation. The direct extraction of the Raman line shape from the CARS data using a maximum entropy method phase retrieval algorithm enables quantitative analysis. The CARS spectra associated with intermediate oxygenation saturation levels can be accurately described by a weighted sum of the fully oxygenated and fully deoxygenated spectra. We find that the degree of oxygenation determined from the CARS data agrees well with that determined by optical absorption. As a nonlinear optical technique, CARS inherently provides the 3-D imaging capability and tolerance to scattering necessary for biomedical applications. We discuss the challenges in extending the proof of principle demonstrated to in vivo applications. (-) 2006 Society of Photo-Optical Instrumentation Engineers. [DOI: $10.1117 / 1.2355671]$

Keywords: blood oxygenation; nonlinear microscopy; coherent antiStokes Raman scattering.

Paper 06137LR received Jun. 7, 2006; revised manuscript received Jul. 3, 2006; accepted for publication Jul. 18, 2006; published online Sep. 21, 2006.

The oxygenation state of the blood is a key determinate of physiological state, and several optically based methods for determining blood oxygenation have been developed for clinical and research use. Many techniques utilize changes in the optical properties of hemoglobin when it binds to molecular oxygen. Intrinsic optical imaging and pulse oxymetry rely on changes in optical absorption, ${ }^{1}$ while Raman techniques utilize changes in specific vibrational modes. ${ }^{2}$ Other methods,

*Tel: +31-20-5256221; E-mail: muller@science.uva.nl such as phosphorescence lifetime quenching, rely on an extrinsic indicator of oxygen tension. ${ }^{3}$ While existing techniques do an excellent job of determining the average oxygenation state of the blood in a tissue, they cannot resolve the signal from individual vessels located within the bulk of a highly scattering tissue. With confocal detection, Raman spectra were obtained from the blood in individual dermal microvessels, but the achievable depth is fundamentally limited to less than $100 \mu \mathrm{m}$ by scattering. ${ }^{4}$ The capability to characterize blood oxygenation on a vessel-by-vessel basis inside optically thick tissue would be a useful capability for biomedical research, enabling detailed studies of neurovascular coupling or oxygen utilization in cancer tumors, for example.

Enabled by recent developments in short-pulse laser technology, coherent anti-Stokes Raman scattering (CARS) microscopy is increasingly being used as a unique microscopic tool. ${ }^{5}$ Since many of the important vibrational spectral features remain resolvable even at room temperature and in samples as complex as live cells, ${ }^{6}$ CARS can potentially provide image contrast with chemical and physical specificity. In addition, because image contrast is produced using a nonlinear optical effect, CARS provides the same capabilities for 3-D imaging inside scattering samples as other nonlinear imaging techniques, such as, for example, two-photon excitation fluorescence microscopy. ${ }^{7}$ The multiplex CARS approach ${ }^{8,9}$ in which a broad-bandwidth femtosecond laser is mixed with a narrow-bandwidth picosecond pulse to generate a CARS signal, provides vibrational spectra with $5-\mathrm{cm}^{-1}$ spectral resolution over a $>200-\mathrm{cm}^{-1}$ spectral range, with an SNR limited only by Poisson noise. ${ }^{10}$ In general, the interpretation of CARS spectra is complicated because of the coherent addition of the different vibrational modes and the nonresonant background. This has limited the use of CARS as a quantitative tool, especially for complex samples. We recently showed, however, that even samples exhibiting highly congested multiplex CARS spectra can be analyzed quantitatively using an approach based on a maximum entropy phase retrieval algorithm. ${ }^{11}$ In earlier work, resonance-enhanced polarizationsensitive coherent Stokes Raman scattering (CSRS) was used to study conformational changes in the heme group of hemoglobin on ligand binding. ${ }^{12}$ Here, we investigate whether multiplex CARS can be used to determine the oxygenation state of hemoglobin, potentially providing a nonlinear optical method for determining blood oxygenation in single vessels deep inside highly scattering tissues.

CARS spectra were obtained using a multiplex CARS setup, which is described in detail elsewhere. ${ }^{13,14}$ Briefly, a 10 -ps [bandwidth $1.5 \mathrm{~cm}^{-1}$ full width at half maximum (FWHM) ] pump/probe laser at $710 \mathrm{~nm}$ is overlapped, in time and space, with an 80-fs (bandwidth $\sim 180 \mathrm{~cm}^{-1}$ FWHM) Stokes laser. The Stokes laser is tunable between 750 and $950 \mathrm{~nm}$, enabling vibrational spectra to be collected in the range of $\sim 750$ to $3500 \mathrm{~cm}^{-1}$. The laser beams are focused with an achromatic lens $(f=5 \mathrm{~cm})$ in a cuvette, which can be translated along the optical axis to measure either a multiplex CARS spectrum of the sample, or-when focused in the glass of the cuvette-of a (nonresonant) reference. Typical laser powers are 17 and $25 \mathrm{~mW}$ for the pump/probe and Stokes lasers, respectively. The generated anti-Stokes signal is fil-

1083-3668/2006/11(5)/050502/3/\$22.00 @ 2006 SPIE 


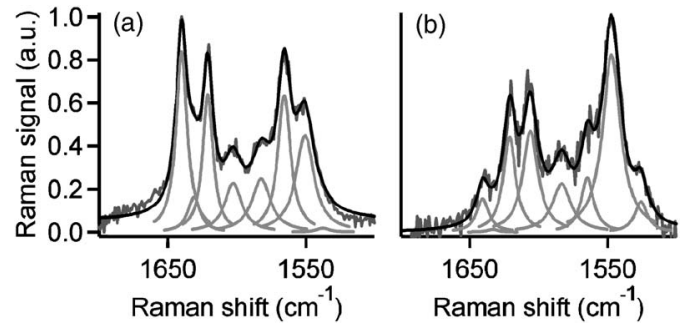

Fig. 1 Normalized Raman spectrum of (a) oxygenated and (b) deoxygenated hemoglobin. The solid black line is a least-squares fit of a sum of Lorentzian contributions to the data. The individual contributions are shown in gray.

tered and spectrally resolved on a spectrometer with an effective spectral resolution of $\sim 5 \mathrm{~cm}^{-1}$. Typical acquisition times for the multiplex CARS spectra are $\sim 1 \mathrm{~s}$. The CARS data are represented as the ratio of the multiplex CARS signal from the sample to that of the reference. This "CARS signal strength" depends only on the strength of the resonant signal relative to the nonresonant background and is independent of experimental parameters, such as laser power or alignment. ${ }^{14}$

Hemoglobin (Instrumentation Laboratory, Lexington, MA) was deoxygenated by exposure to a stream of nitrogen for 1 to $2 \mathrm{~h}$, until dark red. Subsequently the sample was stepwise oxygenated, while stirring continuously, by repeatedly exposing it briefly (20 s) to a stream of $99 \%$ oxygen. After each step, an absorption spectrum was recorded and subsequently either a Raman spectrum or a CARS spectrum was measured. Absorption spectra were recorded on an Uvikon 923 spectrophotometer (Biotek Instruments Inc., Winooski, VT) and Raman spectra were collected using a Raman microscope (Renishaw RM1000, spectral resolution: $\sim 5 \mathrm{~cm}^{-1}$ ) with a $6-\mathrm{mW}$ $\mathrm{HeNe}$ laser, with a typical acquisition time of $25 \mathrm{~min}$.

Raman spectra of oxygenated [Fig. 1(a)] and deoxygenated [Fig. 1(b)] hemoglobin in the vibrational range of 1700 to $1500 \mathrm{~cm}^{-1}$ show significant differences in the vibrational spectrum, notably in the relative intensities of the $\sim 1550$ and $\sim 1565$ and the $\sim 1631$ and $\sim 1640 \mathrm{~cm}^{-1}$ peaks. It is outside the scope of this letter to assign all observed Raman lines to the individual vibrations of the highly complex heme group structure. ${ }^{15}$ Single-component decomposition of a series of Raman spectra at different levels of oxygen saturation (data not shown) reveals that the Raman spectrum for intermediate oxygenation levels can be taken as weighted sum of a fully oxygenated and a fully deoxygenated spectrum.

Multiplex CARS spectra of hemoglobin at different oxygenation states [Fig. 2(a)] clearly show features dependent on the oxygenation state, which is independently determined from optical absorption measurements [Fig. 2(b)]. To quantitatively analyze the multiplex CARS data, direct extraction of the vibrational line shape based on the maximum entropy method $^{11}$ was applied [Fig. 2(c)]. This procedure yields the imaginary part of the third-order nonlinear susceptibility, $\operatorname{Im}\left[\chi^{(3)}\right]$, which can be shown ${ }^{16}$ to be identical to the Raman lineshape if both CARS and Raman spectra are obtained using all-parallel polarization conditions. Comparison of the Raman spectra (Fig. 1) and the $\operatorname{Im}\left[\chi^{(3)}\right]$ spectra [Fig. 2(c)] show similar results, with small differences likely arising from the different electronic resonance conditions produced by the dif-
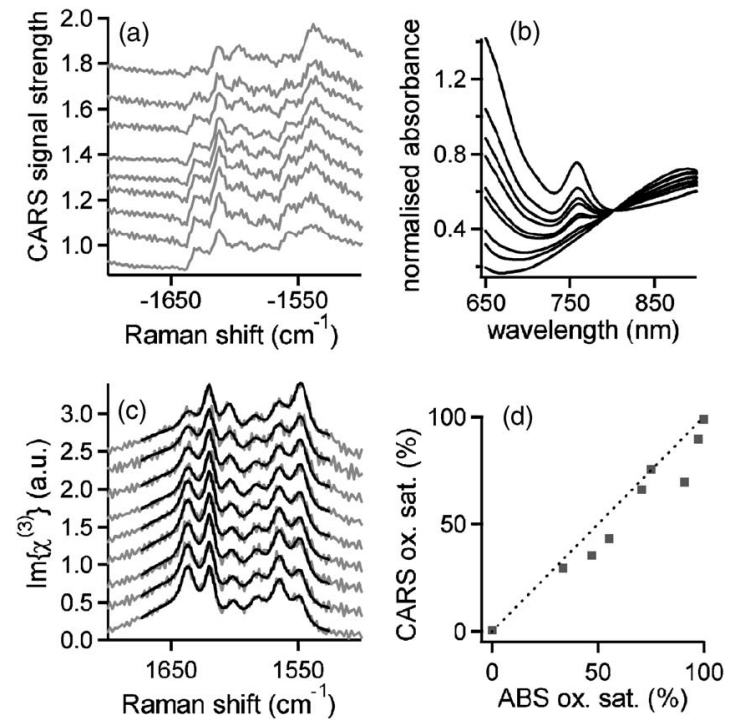

Fig. 2 (a) Multiplex CARS spectra of hemoglobin at different levels of oxygen saturation (increasing from bottom to top), (b) absorption spectra of hemoglobin at different levels of oxygen saturation (decreasing from top to bottom at $650 \mathrm{~nm}$ ), and (c) $\operatorname{Im}\left[\chi^{(3)}\right]$ spectra retrieved from the multiplex CARS spectra of (a). The solid black lines are least-squares fits of the spectra to a weighted sum of fully oxygenated and deoxygenated $\operatorname{Im}\left[\chi^{(3)}\right]$ spectra. The spectra in (a) and (c) are offset for clarity. (d) Oxygen saturation levels determined from the fits of the $\operatorname{Im}\left[\chi^{(3)}\right]$ spectra in (c) versus the level determined from the absorption spectra shown in (b). The dotted line is a guide to the eye indicating a 1:1 correspondence.

ferent wavelengths of excitation (632.8 and $710 \mathrm{~nm}$ for Raman and CARS, respectively).

To obtain a quantitative measure of the level of oxygen saturation from the multiplex CARS spectra, the $\operatorname{Im}\left[\chi^{(3)}\right]$ spectra were fitted with a weighted sum of a fully oxygenated and a fully deoxygenated $\operatorname{Im}\left[\chi^{(3)}\right]$ spectrum [Fig. 2(c)]. The oxygen saturation determined from the ratio of the fit coefficients is nearly identical to the oxygen saturation independently measured using optical absorption [Fig. 2(d)]. As a further demonstration, the oxygen uptake of a fully deoxygenated hemoglobin sample was followed in real time (Fig. 3). The initially deoxygenated sample was continuously stirred and exposed to a stream of $99 \%$ oxygen. Multiplex CARS spectra [Fig. 3(a)] were taken every $2 \mathrm{~s}$ and complete oxygen saturation was reached in $\sim 2 \mathrm{~min}$. The oxygenation state as a function of time was then quantified by fitting the $\operatorname{Im}\left[\chi^{(3)}\right]$ spectra retrieved from the CARS data [Figs. 3(b) and 3(c)] to a weighted sum of the fully oxygenated and deoxygenated $\operatorname{Im}\left[\chi^{(3)}\right]$ spectra [Fig. 3(d)].

The experimental results presented here demonstrate that multiplex CARS can be used to quantitatively measure the oxygen saturation of hemoglobin, with oxygenation values that agree well with those determined from optical absorption (Fig. 2). In addition, the $\sim 1$-s signal acquisition time enables dynamic processes to be followed in time (Fig. 3) and, because the signal arises from a nonlinear optical process, from a 3-D localized region in the sample. We anticipate that our technique will provide a maximum penetration depth comparable to that of imaging techniques based on other nonlinear optical processes, such as two-photon excited fluorescence, 

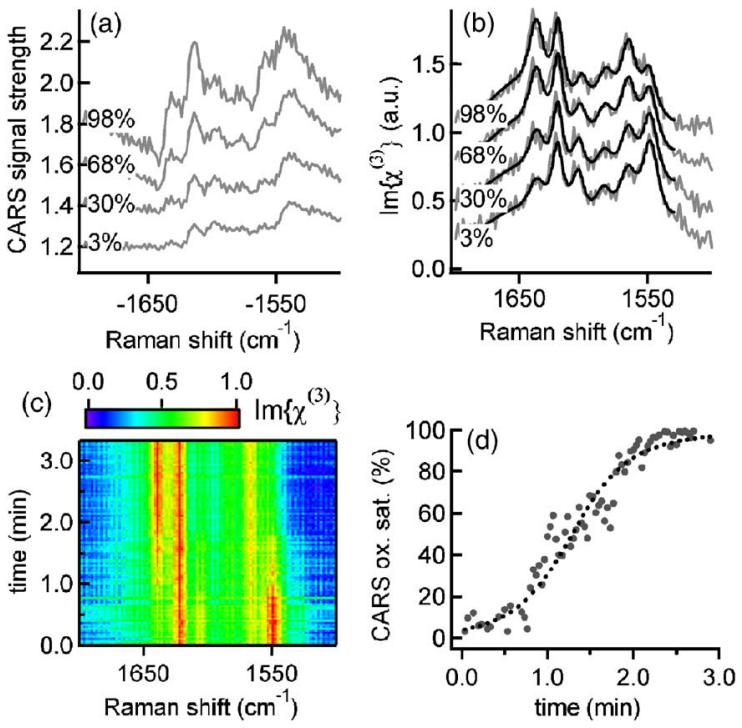

Fig. 3 Representative CARS (a) and derived $\operatorname{Im}\left[\chi^{(3)}\right]$ spectra (b) from a set of measurements following the oxygenation of a hemoglobin sample. The solid black lines in (b) are least-squares fits of the spectra to a weighted sum of fully oxygenated and deoxygenated $\operatorname{Im}\left[\chi^{(3)}\right]$ spectra. The derived levels of oxygenation saturation are indicated with the spectra, which are offset for clarity. (c) Image representation of the complete set of measured $\operatorname{Im}\left[\chi^{(3)}\right]$ spectra as a function of time and (d) level of oxygenation saturation as a function of time determined from a least-squares fits-analogous to that of (b) - of the $\operatorname{Im}\left[\chi^{(3)}\right]$ data of (c). The dotted line is a guide to the eye.

i.e., in excess of $0.5 \mathrm{~mm}$ in brain tissue. The nonlinear nature of CARS provides inherent sectioning capability, since it ensures that any CARS light detected, scattered or not, must have originated in the high-intensity focal volume, also alleviating scattering-induced degradation of the resolution.

The laser wavelengths used in this study have been shown ${ }^{15}$ to be nearly ideal for the study of whole blood since they yield Raman spectra that are free from the strong Raman scattering from carotenoids and protein bands present with shorter wavelength excitation. Nonetheless, extension of this work to in vivo situations will first require validation of the method in whole blood. Another concern for in vivo work is the effect of highly scattering samples on the detection scheme. The CARS signal is dominantly generated in the propagation direction of the excitation lasers, often impossible to directly detect for in vivo samples. Recent work, however, has shown that backscattering of CARS signal generated in the focal volume by deeper-lying tissue results in a substantial signal in the backward direction. ${ }^{17}$ Since the changes in the vibrational spectrum on the oxygenation state are quite significant, scattering-induced spectral blurring is not expected to be a major problem. Although the fluence levels employed here approach the damage threshold for biological samples under sustained illumination, the flow of the blood, coupled with high-numerical-aperture optics, should mitigate any damage.

Once extended to in vivo systems, the approach presented here may provide a unique capability in biomedical research. For example, one could characterize the change in blood oxygenation in individual brain capillaries in response to local neural activity, using anesthetized rodents with a craniotomy that provides optical access to the brain. Such experiments would refine our understanding of the additional metabolic load associated with increased neural firing, and would help to further elucidate the contrast mechanisms in blood-oxygenlevel-dependent functional magnetic resonance imaging, and to connect that contrast to the underlying neural activity.

\section{Acknowledgments}

We thank Prof. G. J. Brakenhoff for initiating this project. Preliminary experiments were performed at the CARS Workshop run by Prof. Sunney Xie at Harvard University with the help of Conor Evans. This work is part of the research program of the Stichting voor Fundamenteel Onderzoek der Materie (FOM), which is financially supported by the Nederlandse Organisatie voor Wetenschappelijk Onderzoek (NWO).

\section{References}

1. A. Grinvald, E. Lieke, R. D. Frostig, C. D. Gilbert, and T. N. Wiesel, "Functional architecture of cortex revealed by optical imaging of intrinsic signals," Nature (London) 324(6095), 361-364 (1986).

2. I. P. Torres Filho, J. Terner, R. N. Pittman, L. G. Somera III, and K. R. Ward, "Hemoglobin oxygen saturation measurements using resonance Raman intravital microscopy," Am. J. Physiol. Heart Circ. Physiol. 289, 488-495 (2005).

3. I. Vanzetta and A. Grinvald, "Increased cortical oxidative metabolism due to sensory stimulation: implications for functional brain imaging," Science 286(5444), 1555-1558 (1999).

4. P. J. Caspers, G. W. Lucassen, and G. J. Puppels, "Combined in vivo confocal Raman spectroscopy and confocal microscopy of human skin," Biophys. J. 85(1), 572-580 (2003).

5. A. Volkmer, "Vibrational imaging and microspectroscopies based on coherent anti-Stokes Raman scattering microscopy," J. Phys. D 38, R59-R81 (2005).

6. G. J. Puppels, F. F. M. de Mul, C. Otto, J. Greve, M. Robert-Nicoud, D. J. Arndt-Jovin, and T. M. Jovin, "Studying single living cells and chromosomes by confocal Raman microspectroscopy," Nature (London) 347, 301-303 (1990).

7. W. Denk, J. H. Strickler, and W. W. Webb, "Two-photon laser scanning fluorescence microscopy," Science 248, 73-76 (1990).

8. M. Müller and J. M. Schins, "Imaging the thermodynamic state of lipid membranes with multiplex CARS microscopy," J. Phys. Chem. $B$ 106(14), 3715-3723 (2002).

9. J. Cheng, A. Volkmer, L. D. Book, and X. S. Xie, "Multiplex coherent anti-Stokes Raman scattering microspectroscopy and study of lipid vesicles," J. Phys. Chem. B 106(34), 8493-8498 (2002).

10. G. W. H. Wurpel, J. M. Schins, and M. Müller, "Direct measurement of chain order in single lipid mono- and bilayers with multiplex CARS," J. Phys. Chem. B 108(11), 3400-3403 (2004).

11. E. M. Vartiainen, H. A. Rinia, M. Bonn, and M. Müller, "Extraction of Raman line-shapes from congested CARS spectra," Opt. Express 14(8), 3622-3630 (2006).

12. A. Voroshilov, G. W. Lucassen, C. Otto, and J. Greve, "Polarizationsensitive resonance CSRS of deoxy- and oxyhaemoglobin," J. Raman Spectrosc. 26(6), 443-450 (1995).

13. G. W. H. Wurpel, H. A. Rinia, and M. Müller, "Imaging orientational order and lipid density in multilamellar vesicles with mulitplex CARS microscopy," J. Microsc. 218(1), 37-45 (2005).

14. H. A. Rinia, M. Bonn, and M. Müller, "Quantitative multiplex CARS spectroscopy in congested spectral regions," J. Phys. Chem. B 110(9), 4472-4479 (2006).

15. H. Sato, H. Chiba, H. Tashiro, and Y. Ozaki, "Excitation wavelengthdependent changes in Raman spectra of whole blood and hemoglobin:comparison of the spectra with 514.5-, 720-, and 1064-nm excitation," J. Biomed. Opt. 6(3), 366-370 (2001).

16. E. M. Vartiainen, "Phase retrieval approach for coherent anti-Stokes Raman scattering spectrum analysis," J. Opt. Soc. Am. B 9(8), 12091215 (1992)

17. C. L. Evans, E. O. Potma, M. Puoris'haag, D. Cote, C. P. Lin, and X. S. Xie, "Chemical imaging of tissue in vivo with video-rate coherent anti-Stokes Raman scattering microscopy," Proc. Natl. Acad. Sci. U.S.A. 102(46), 16807-16812 (2005). 